\title{
Hyperglycemic Crises in Patients with Covid-10
}

Nasser Mikhail*, Soma Wali

Department of Medicine, OliveView-UCLA Medical Center, Sylmar, CA 91342, United States

*Corresponding author: Nasser Mikhail, Department of Medicine, OliveView-UCLA Medical Center, Sylmar, CA 91342, United States Received date: August 01, 2020; Accepted date: September 01, 2020; published date: September 16, 2020

Citation: Nasser Mikhail. Hyperglycemic Crises in Patients with Covid-10. J Clinical Research and Reports, 5(4); DOI:10.31579/2690-1919/114 Copyright: () 2020 Nasser Mikhail. This is an open access article distributed under the Creative Commons Attribution License, which permits unrestricted use, distribution, and reproduction in any medium, provided the original work is properly cited.

\section{Abstract}

Background: It is unclear whether the 2 hyperglycemic crises, diabetic ketoacidosis (DKA), and hyperosmolar hyperglycemic state (HHS) have different characteristics in patients with COVID-19.

Objective: to describe prevalence, outcomes, and management of hyperglycemic crisis specifically in patients with COVID-19.

Methods: English literature search of electronic databases supplemented by manual search up to July $31^{\text {st }}$, 2020. Search terms included hyperglycemic crises, diabetic ketoacidosis, COVID-19, ARDS, dexamethasone, mortality, and safety. Since no randomized trials are available, all pertinent observational studies, case reports and major organization guidelines were reviewed.

Results: DKA occurs in 0.45 to $3.4 \%$ of patients with COVID-19 admitted to the hospital, and results in approximately $50 \%$ mortality rate. Excessive intravenous hydration should be avoided in patients at risk or having acute respiratory distress syndrome (ARDS) to avoid volume overload. In patients presenting with hyperglycemic crisis and COVID-19 requiring oxygen or on mechanical ventilation, dexamethasone may be given after resolution of hyperglycemic crisis. Insulin doses need to be increased by $50-100 \%$ to control dexamethasone-induced hyperglycemia. Selected patients with non-complicated both DKA and COVID19 may be safely managed by subcutaneous rapid-acting insulin in a step-down unit with blood glucose monitoring every 2 hours. This strategy may spare beds in the intensive care unit (ICU) and personal protective equipment (PPE), and decrease nursing time at bedside.

Conclusions: Hyperglycemic crises with COVID-19 are uncommon but carry high mortality rate. Uncomplicated cases may be managed ia step-down unit. Dexamethasone can be given after resolution of hyperglycemic crisis.

Key words: hyperglycemic crises; diabetic ketoacidosis COVID-19; ARDS; dexamethasone; mortality; safety; intensive care unit

\section{Introduction}

Hyperglycemic crises include DKA and HHS. DKA is the most common hyperglycemic emergency and occurs more commonly in patients with type 1 diabetes [1]. Approximately one third of cases of DKA occurs in type 2 diabetes [1]. In nearly $50 \%$ of patients with type 2 diabetes, DKA is the initial manifestation of diabetes [1]. It is not uncommon that DKA and HHS coexist in the same patient. In a large retrospective study of 1,211 patients with hyperglycemic crisis in Atlanta area, Georgia, $38 \%$ of patients had isolated DKA, 35\% had isolated HHS, and $27 \%$ had combined features of DKA and HHS [2].] The purpose of this article is to review the prevalence, outcomes, and management of patients with COVID-19 complicated by hyperglycemic crisis based on available studies, and authors' experience.

\section{Prevalence of hyperglycemic crises in COVID-19}

Available studies suggest that DKA is the most common of hyperglycemic crises among patients with COVID-19. In the CORONADO French study, the largest available study dedicated to patients with COVID-19 and diabetes $(n=1,317), 19$ patients $(1.4 \%)$ presented with DKA [3]. In one retrospective study from UK, 4 of 218 patients $(1.8 \%)$ admitted with COVID-19 presented with DKA [4]. In another UK series, 8 of 232 patients (3.4\%) with COVID-19 had DKA [5]. In one retrospective study from Wuhan, China, including 658 patients with COVID-19 admitted to the hospital, only 3 patients $(0.45 \%)$ had DKA [6]. Therefore, available data suggests that prevalence of DKA associated with COVID-19 is not common and ranges from $0.45 \%$ to $3.4 \%$ [3-6].

Although DKA occurs more commonly in type 1 diabetes [1], most (80$88 \%$ ) of the episodes of hyperglycemic crises in patients with COVID-19 occurred in patients with type 2 diabetes $[7,8]$. Mixed DKA and HHS features were reported in few studies. In a retrospective study from UK, Armeni et al [7] described 26 patients with hyperglycemic crisis and COVID-19; 13 patients (50\%) had combined features of DKA and HHS, 11 patients $(42 \%)$ had isolated DKA, and 2 patients (7\%) had isolated HHS. Chan et al [9] reported a series of 6 men (age range 19-62 years) with COVID-19 presenting with a mixed picture of DKA and HHS.

\section{Outcomes of patient with COVID-19 and hyperglycemic crisis}

Latest available data in the USA showed that mortality in DKA patients has dropped to $0.4 \%$ [10]. Mortality in patients with COVID-19 complicated by DKA appears to be much higher reaching $50 \%$ in most series $[7,8]$. This finding is most likely due to older age, co-morbidities, 
and increase disease burden by COVID-19 itself. In the largest reported series of 50 patients in Bronx area (New York) admitted with DKA and COVID-19, 25 (50\%) died [8]. Likewise, among the 4 patients described by Goldman [4], 2 patients died, and one patient remained in critical unit at the end of 30 days follow-up. Unexpectedly, in the series reported by Akundo et al [5] in UK, COVID-19 patients with DKA were more likely to survive $(87.1 \%)$ compared with patients without DKA $(50.6 \%) \mathrm{P}=$ 0.046 . The reasons of the latter finding are unclear, but may be related to differences in patients' characteristics, co-morbidities, and severity of COVID-19.

Mortality data regarding patients with COVID-19 complicated by isolated HHS are not available. Meanwhile, in patients with diabetes but without COVID-19 patients, it was shown that mortality was higher when DKA and HHS coexist compared with each condition alone [2]. This observation is in agreement with outcomes of the 6 patients with mixed DKA/HHS reported by Chan et al [9], of whom 4 patients expired.

\section{Mechanisms of hyperglycemic crisis in the setting COVID-19}

It is well-known that any type of infection can precipitate hyperglycemic crisis, particularly respiratory infection such as COVID-19 [11,12]. In addition, there are 2 potential mechanisms whereby COVID-19 may trigger hyperglycemic crisis. First, angiotensin-converting enzyme 2 (ACE 2) functions as the receptor of the virus causing COVID-19 (the severe acute respiratory syndrome coronavirus 2, abbreviated as SARSCoV-2) and the virus SARS-CoV-1 responsible for the SARS epidemic in 2002 to 2004 [13]. In addition to its presence in the lungs, ACE2 is also expressed in the $\beta$ cells of pancreas [14]. Thus, binding of the SARSCoV-2 to ACE 2 may virtually cause damage of pancreatic $\beta$-cells and cause acute diabetes [14]. Second, ACE 2 catalyzes the conversion of angiotensin II to angiotensin 1-7 [13]. The binding of SARS-CoV-2 to ACE 2 down regulates ACE 2 and lead to increased levels of angiotensin II [15]. The latter may decrease insulin secretion and virtually contribute to the development of hyperglycemic crisis [16].

\section{Factors to be considered in management of patients with COVID-19 and hyperglycemic crisis.}

The management of hyperglycemic crisis has not significantly changed in last 30 years, and consists of intravenous fluids, insulin, and potassium [11]. No specific guidelines are available for management of hyperglycemic crisis in patients with COVID-19. However, there are some important issues specific to COVID-19 complicated by hyperglycemic crises discussed below.

\section{Patients with ARDS}

ARDS is a common complication of COVID-19 ranging from $41 \%$ among all patients admitted to the hospital to $71 \%$ of patients admitted to the ICU $[17,18]$. If patient has DKA or HHS on top on ARDS due to COVID-19, it is recommended to avoid aggressive hydration, and to use intravenous fluids judiciously to avoid volume overload and worsening pulmonary edema [7,15].

\section{Patients requiring oxygen or mechanical ventilation}

Data from the RECOVERY trial showed that dexamethasone $6 \mathrm{mg} / \mathrm{d}$ for up to 10 days decreased 28-day mortality in patients with COVID-19 requiring oxygen or invasive mechanical ventilation, but not in patients not requiring oxygen [19].The highest magnitude of mortality reduction was observed among patients receiving invasive mechanical ventilation, adjusted rate ratio (RR) $0.64,95 \% \mathrm{CI}$; 0.51 to 0.81 (P < 0.001), followed by patients receiving oxygen without mechanical ventilation, RR 0.82 , 95\% CI, 0.72 to $0.94(\mathrm{P}=0.002)$ [19]. In the RECOVERY trial, $24 \%$ of patients had diabetes at the study entry [19]. Unfortunately, no data were reported regarding the effect of dexamethasone on mortality among the diabetic subgroup. In the meantime, it is well established that glucocorticoids such as dexamethasone worsen hyperglycemia and may even precipitate DKA [20]. Nonetheless, given the substantial mortality benefit achieved by dexamethasone therapy, and the relatively low-dose used, it is worthwhile to offer dexamethasone to appropriate patients with COVID-19 having DKA or HHS. However, it may be wise to start dexamethasone after the resolution of hyperglycemic crisis, which usually takes $24-48$ hours. The authors recommend increasing insulin doses by $50-100 \%$ within 4 hours after administration of dexamethasone to avoid rebound in hyperglycemia. Fortunately, the mortality benefit in patients with COVID-19 was evident in relatively late stage of COVID-19. Thus, pre-specified subgroup analysis of the RECOVERY trial showed that dexamethasone was associated with reduction in 28-day mortality among patients with symptoms for more than 7 days but not among those with more recent symptom onset [19]. Thus, this delay may allow time for full recovery of the hyperglycemic crisis.

\section{Patients with euglycemic DKA}

DKA presenting with relatively low blood glucose levels $(<250 \mathrm{mg} / \mathrm{dl})$ may uncommonly occur as adverse effects of sodium-glucose cotransporters type 2 (SGLT-2) inhibitors approved for treatment of type 2 diabetes [21]. Few case reports described patients with COVID-19 treated with SGLT-2 inhibitors presenting with euglycemic DKA [7,22]. Clearly, SGLT2 inhibitors should be discontinued and not resumed in the future. Treatment of euglycemic DKA follows the same protocol of classic DKA. However, if the presenting plasma glucose concentrations are less than $200 \mathrm{mg} / \mathrm{dl}$, early addition of D5 or D10 to intravenous fluids is recommended to allow continuing insulin administration while preventing hypoglycemia.

\section{Need for sparing ICU beds and PPE}

During the current pandemic crisis of COVID-19, there is great need for every ICU bed for management of critically-ill patients requiring mechanical ventilation. Likewise, in many places, there is shortage of PPE Several randomized trials have shown that uncomplicated cases of DKA can be safely and effectively managed outside the ICU in step-down unit using rapid-acting subcutaneous insulin (lispro or aspart) every $1-2 \mathrm{~h}$ [23-25]. Duration of time until resolution of DKA, frequency of hypoglycemia, and length of hospital stay did not differ between patients who received subcutaneous insulin compared with those who received intravenous insulin infusion [23-26]. Moreover, in one study, there was $39 \%$ cost saving with the use of subcutaneous insulin [25]. Interestingly, with respect to the frequency of insulin injection, no differences in the above-mentioned outcomes were demonstrated when rapid-acting insulin aspart was given subcutaneously every 2 hours compared to every hour or to intravenous insulin infusion [24]. Furthermore, blood glucose levels were checked every 2 hours by fingerstick in the group of patients randomized to subcutaneous insulin every 2 hours [24]. Implementation of the latter strategy may be useful to reduce the frequency of patient interactions and need for PPE [26,27]. It should be emphasized, however, that only uncomplicated cases of DKA or HHS may be managed outside the ICU. The definition of uncomplicated cases is not well-defined in the literature. In studies using subcutaneous insulin to treat DKA, the following exclusion criteria were applied: refractory hypotension, acute myocardial ischemia, end-stage renal and liver disease, anasarca, and pregnancy [23-25]. The authors would like to add the following exclusion criteria: altered mental status, and any organ failure due to the concomitant COVID-19 such as respiratory failure (e.g. hypoxia, severe tachypnea). In any case, clinical judgement should be taken on an individual basis with final decision to be taken by the treating medical team. 


\section{Conclusions and future needs}

While hyperglycemic crises, mainly DKA, occur in less than $3.5 \%$ of patients with COVID-19 admitted to the hospital, they are associated with approximately $50 \%$ mortality. In general, treatment of hyperglycemic crises in the setting of COVID-19 is similar to non-COVID-19 patients. However, less aggressive intravenous fluid is indicated in patients with ARDS to avoid volume overload. Dexamethasone may be given to patients requiring oxygen or invasive mechanical ventilation after the resolution of hyperglycemic crisis as it decreases mortality in these patients. Meanwhile, insulin doses should be increased by 50 to $100 \%$ within few hours after starting dexamethasone to counteract its hyperglycemic effect. Carefully selected patients with non-complicated both COVID-19 and DKA may be managed in step-down unit with subcutaneous insulin administered every 2 hours. This strategy may spare ICU beds, PPE, and reduce nursing time at bedside. Randomized studies are urgently needed to determine the optimum management of hyperglycemic crisis in association with COVID-19. Specifically, these trials should define what is the safest type and rate of infusion of intravenous fluid, ideal protocol for insulin administration, and optimum glycemic targets.

\section{Conflict of interest}

The authors have no conflict of interest to declare.

\section{References}

1. Nyenwe EA, Kitabchi AE. The evolution of diabetic ketoacidosis: An update of its etiology, pathogenesis and management. Metabolism 2016; 65: 507-521.

2. Pasquel FJ, Tsegka $\mathrm{K}$, Wang $\mathrm{H}$ et al. Clinical outcomes in patients with isolated or combined diabetic ketoacidosis with isolated or combined diabetic ketoacidosis and hyperosmolar hyperglycemic state: a retrospective, hospital-based cohort study. Diab Care 2020; 43(2): 349-357.

3. Cariou B, Hadjadji S, Wargny M, et al, for the CORONADO investigators. Phenotypic characterization and prognosis of inpatients with COVID-19 and diabetes: the CORONADO study. Diabetologia 2020; Epub ahead of print.

4. Goldman N, Fink D, Cai J, et al. High prevalence of COVID19-associated diabetic ketoacidosis in UK secondary care. Diab Res Clin Pract 2020; 166: 108291.

5. Alkundi A, Mahmoud I, Musa A, et al. Clinical characteristics and outcomesof COVID-1 hospitalized patients with diabetes in the United Kingdom: a retrospective single centre study. Diab Res Clin Pract 2020; Epubahead of print 2020; published June 10.

6. Li J, Wang X, Chen J, et al. COVID-19 infection may cause ketosis and ketoacidosis. Diab Obes Metab 2020; Epub ahead of print. Published April 20.

7. Armeni E, Aziz U, Qamar S, et al. Protracted hyperketonemia in hyperglycaemic emergencies in COVID-19: a retrospective case series. Lancet Diab Endocrinol 2020; 8: 660-663.

8. Chamarro-Pareja N, Parthasarathy $\mathrm{S}$, Annam $\mathrm{J}$, et al. Unexpected high mortality in COVID-19 and diabetic ketoacidosis. Metabolism 2020. E pub ahead of print. Published June 24.

9. Chan KH, Thimmareddygari D, Rmahi A, et al. Clinical characteristics and outcome in patients with combined diabetic ketoacidosis and hyperosmolar hyperglycemic state associated with COVID-19: a retrospective, hospital-based observational case series. Diab Res Clin Pract 2020; 166: 108279.

10. Benoit SR, Zhang W, Geiss LS, et al. Trends in diabetic ketoacidosis hospitalizations and in-hospital mortality- United
States, 2000-2014. MMWR Morb Mort Wkly Rep 2018; 67 (12): 362-365.

11. Kitabchi AE, Umpierrez GE, Miles JM, Fisher JN. Hyperglycemic crises in adult patients with diabetes. Diab Care 2009; 32 (7): 1335-1343.

12. Stratigou T, Vallianou N, Vlassopoulo B, et al. DKA cases over the last three years: has anything changed? Diab Metab Syndr 2019; 13: 1639-1641.

13. Vaduganathan $M$, Vardeny $\mathrm{O}$, Michel $\mathrm{T}$, et al. ReninAngiotensin-Aldosterone system inhibitors in pastients with Covid-19. N Engl J Med 2020; 382: 1653-1659.

14. Yang JK, Lin SS, Ji XJ, Guo LM. Binding of SARS coronavirus to its receptor damages islets and causes acute diabetes. Acta Diabetol 2010; 47(3): 193-199.

15. Chee YJ, Ng SJH, Yeoh E. Diabetic ketoacidosis precipitated by Covid-19 in a patient with newly diagnosed diabetes mellitus. Diab Res Clin Pract 2020; 164: 108166.

16. Fliser D, Schaefer F, Schmid D, et al. Angiotensin II affects basal, pulsatile, and glucose-stimulated insulin secretion in humans. Hypertension 1997; 30 (5): 1156-1161.

17. Wu C, Chen X, Cai Y, et al. Risk factors associated with acute respiratory distress syndrome and death in patients with coronavirus disease 2019 pneumonia in Wuhan, China. JAMA Intern Med 2020; e200994, epub ahead of print.

18. Yu Y, Xu D, Fu S, et al. Patients with COVID-19 in 19 ICUs in Wuhan, China: a cross-sectional study. Crit Care 2020; 24: 219.

19. The RECOVERY Collaborative Group. Dexamethasone in hospitalized patients with Covid-19 - Preliminary Report N Engl J Med 2020; Epub ahead of print. Published July 17.

20. Rahman SA, Karmakar A, Almustafa MM. Preoperative steroids triggering ketoacidosis in the neurosurgical patient. J Clin Anesth 2018; 46: 33-34.

21. Rosenstock J, Ferrannini E, Euglycemic diabetic ketoacidosis: a predictable detectable, and preventable safety concern with SGLT2 inhibitors. Diab Care 2015; 38: 21.1638-1642.

22. Oriot P, Hermans MP. Euglycemic diabetic ketoacidosis in a patient with type 1 diabetes and SARS-Cov-2 pneumonia case report and review of the literature. Acta Clin Belg 2020; Epub Ahead of print June 16.

23. Cohn BG, Keim SM, Watkins JM, Camargo CA. Does management of diabetic ketoacidosis with subcutaneous rapidacting insulin reduce the need for intensive care unit admission? J Emerg Med 2015; 49 (4): 530-537.

24. Umpierrez GE, Cuervo R, Karabell A, et al. Treatment of diabetic ketoacidosis with subcutaneous insulin aspart. Diab Care 2004; 27 (8): 1873-1878.

25. Umpierrez GE, Latif K, Stoever J, et al. Efficacy of subcutaneous insulin lispro versus continuous intravenous regular insulin for the treatment of patients with diabetic ketoacidosis. Am J Med 2004; 117: 291-296.

26. Korytkowski M, Antinori-Lent K, Drincic A, et al. Pragmatic approach to inpatient diabetes management during the COVID19 pandemic. J Clin Endocrinol Metab 2020; 105 (9): dgaa342.

27. Palermo NE, Sadhu AR, McDonnell ME. Diabetic ketoacidosis in COVID-19: unique concerns and considerations. J Clin Endocrinol Metab 2020; 105 (8): 1-11. 